\title{
Designing a gel-fiber composite to extract nanoparticles from solution
}

\section{Citation}

Liu, Ya, Xin Yong, Gerald McFarlin, Olga Kuksenok, Joanna Aizenberg, and Anna C. Balazs. 2015. "Designing a Gel-fiber Composite to Extract Nanoparticles from Solution." Soft Matter 11 (44): 8692-8700. doi:10.1039/c5sm01719j.

\section{Published Version}

doi:10.1039/C5SM01719J

\section{Permanent link}

http://nrs.harvard.edu/urn-3:HUL.InstRepos:37255075

\section{Terms of Use}

This article was downloaded from Harvard University's DASH repository, and is made available under the terms and conditions applicable to Open Access Policy Articles, as set forth at http:// nrs.harvard.edu/urn-3:HUL.InstRepos:dash.current.terms-of-use\#OAP

\section{Share Your Story}

The Harvard community has made this article openly available.

Please share how this access benefits you. Submit a story.

\section{Accessibility}




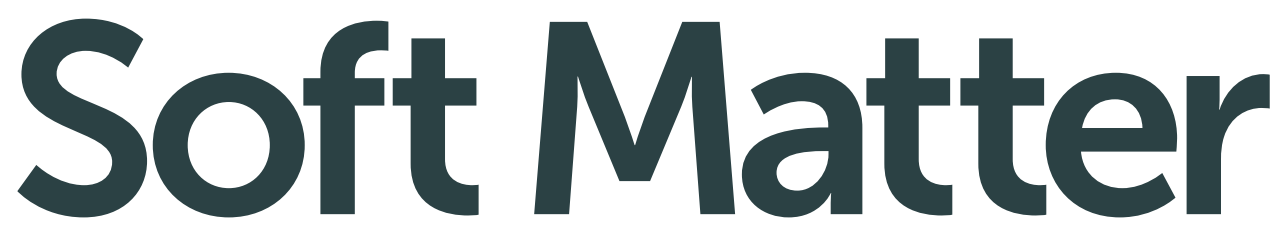

Accepted Manuscript

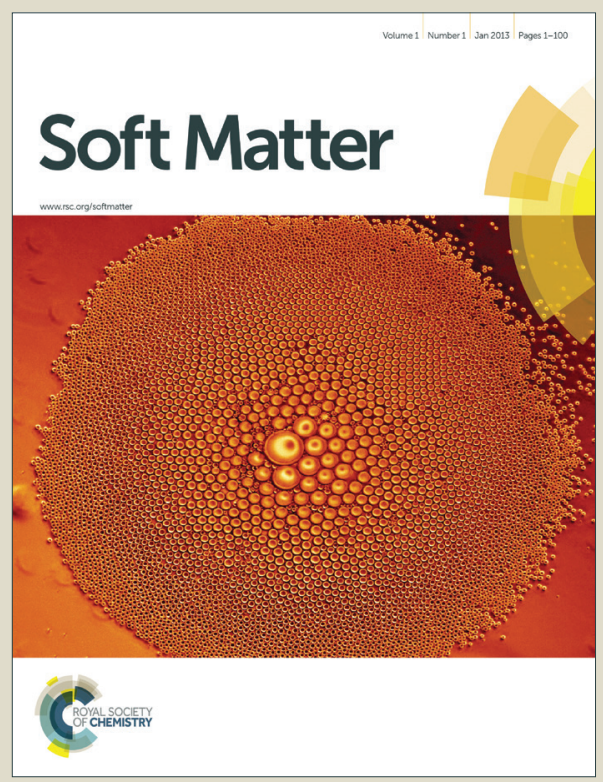

This is an Accepted Manuscript, which has been through the Royal Society of Chemistry peer review process and has been accepted for publication.

Accepted Manuscripts are published online shortly after acceptance, before technical editing, formatting and proof reading. Using this free service, authors can make their results available to the community, in citable form, before we publish the edited article. We will replace this Accepted Manuscript with the edited and formatted Advance Article as soon as it is available.

You can find more information about Accepted Manuscripts in the Information for Authors.

Please note that technical editing may introduce minor changes to the text and/or graphics, which may alter content. The journal's standard Terms \& Conditions and the Ethical guidelines still apply. In no event shall the Royal Society of Chemistry be held responsible for any errors or omissions in this Accepted Manuscript or any consequences arising from the use of any information it contains. 


\title{
Designing a Gel-Fiber Composite to Extract Nanoparticles from Solution
}

Ya Liu, Xin Yong, ${ }^{\dagger}$ Gerald McFarlin, IV, Olga Kuksenok, Joanna Aizenberg, ${ }^{\S}$ Anna C. Balazs* Chemical Engineering Department, University of Pittsburgh, Pittsburgh, PA 15261

†Department of Mechanical Engineering, Binghamton University, State University of New York, Binghamton, NY, 13902-6000

${ }^{\S}$ Wyss Institute for Biologically Inspired Engineering, Department of Chemistry and Chemical Biology, and School of Engineering and Applied Science, Harvard, Cambridge, MA, 02138

\begin{abstract}
The extraction of nanoscopic particulates from flowing fluids is a vital step in filtration processes, as well as the fabrication of nanocomposites. Inspired by the ability of carnivorous plants to use hair-like filaments to entrap species, we use computational modeling to design a multi-component system that integrates compliant fibers and thermo-responsive gels to extract particles from the surrounding solution. In particular, hydrophobic fibers are embedded in a gel that exhibits a lower critical solution temperature (LCST). With an increase in temperature, the gel collapses to expose fibers that self-assemble into bundles, which act as nanoscale "grippers" that bind the particles and draw them into the underlying gel. By varying the relative stiffness of the fibers, the fiber-particle interaction strength and the shear rate in the solution, we identify optimal parameters where the particles are effectively drawn from the solution and remain firmly bound within the gel layer. Hence, the system can be harnessed in purifying fluids and creating novel hybrid materials that integrate nanoparticles with polymer gels.
\end{abstract}

\section{* corresponding author e-mail: balazs@pitt.edu}




\section{Introduction}

Biological organisms often employ stealth mechanisms to trap their prey. Such complexity of behavior is also apparent in the plant world. Consider, for example, the behavior of the Venus fly trap. ${ }^{1}$ Insects are lured to these plants, but once they land on the leaves, hidden hairs trap these bugs and ensure that they cannot escape. This structural motif provides useful design rules for creating synthetic systems that can extract and effectively trap particulates from a solution, and hence, remove solutes from the fluid. Recent experimental studies have in fact demonstrated that synthetic hair-like structures can be used to manipulate a range of particles in solution. ${ }^{2,3}$ Here, we use computational modeling to devise a system where fibers embedded in a thermo-responsive gel act as stealth-like hairs to entrap particles in a flowing fluid. In particular, the fibers remain hidden within the gel layer until the temperature of the system is increased. Once exposed, the fibers can extend into the solution to bind and "grip" adhesive particles, drawing them into the underlying gel layer. The combination of the fibers and the gel layer inhibits the particle from escaping the surface and returning into the solution. Thus, the system could be used as an effective filtration device, cleaning the surrounding solution from contaminants. Alternatively, the approach could be used to draw nanoparticles in solution into the gel layer, and thereby, provide an effective means of creating novel gel nanocomposites.

To model this system, we use a coarse-grained particle-based computational method known as dissipative particle dynamics (DPD). Figure 1 shows the initial configuration of the different components in the DPD simulation. The fibers are localized in a thermo-responsive gel and both fibers and gel are anchored to a solid substrate. These fibers are formed from a single strand of beads and have a sufficiently high persistence length that they are relatively stiff objects (see Methodology). Moreover, the fibers are not chemically attached to gel, and the fiber- 
gel interaction is relatively incompatible. Notably, there are 30 beads between the cross-linkers in this tetra-functional polymer network. Given that the fibers are only a single bead in width and the relative sparsity of the polymer network, each fiber is surrounded by a significant volume of just solvent beads.

The gel exhibits a lower critical solution temperature (LCST); hence, it collapses when the temperature, $T$, is increased and expands when $T$ is decreased. As the starting point for simulation, the gel layer covers the fibers, which are aligned in the vertical direction (Fig. 1a.) We then exploit the collapse of the gel with an increase in $T$. Due to the stiffness of the fibers, the large ratio between the mesh size of the gel and the diameter of the fiber, as well as the fact that the fibers are not chemically bound to the polymer network, the fibers do not collapse as the gel shrinks. Rather, as we show below, the temperature-dependent contraction of the gel exposes the fibers to the host solution. Since the fibers are taken to be incompatible with the fluid, they are driven to bunch together and can effectively act as "grippers" for particles in the solvent. The particle is also solvophobic and displays a relative attraction to the fibers. Under these conditions, these fibers can wrap around the particle and draw it toward the gel-coated lower surface, thereby removing it from the solution. The particles in this simulation can represent nanoscopic impurities, and thus, the system would be effective at removing pollutants from the fluid.

As noted above, the system is useful in another context: creating gel-nanoparticle composite coatings. Consider for example, the case where the solid spheres in Fig. 1 represent gold nanoparticles; then the synergistic interactions between the fibers and thermo-responsive gel provides an effective means of extracting the gold from the solution and localizing the particles in the collapsed polymeric layer to form a hybrid film, which could display useful 
plasmonic properties. ${ }^{4}$ In effect, with the increase in temperature, the exposed fibers provide the "hook" by which the nanoparticles are successfully caught and embedded within the polymer network. The concomitant collapse of the LCST yields a dense polymer matrix, and thus, a vital component for the nanocomposite coating. Notably, gel nanocomposites are relatively new materials and designing effective modes of fabrication can facilitate their development for a range of applications. ${ }^{5}$

Herein, we examine the factors that affect the ability of the fibers to bind the particles and bring them into the gel layer, particularly focusing on the roles that the relative stiffness of the fiber and the fiber-particle interaction strength play in this function. Below, we first describe the DPD approach used in our studies and then discuss the behavior of this multi-component system.

\section{Methodology}

Our system is depicted in Fig.1a, which displays the array of relatively stiff fibers (white beads) that are anchored to the substrate (brown beads). The fibers are embedded in a thermoresponsive hydrogel (green beads) that exhibits lower critical solution temperature (LCST) behavior, and thus, swells at lower temperatures, $T$, and collapses at higher $T$. In the initial configuration, the spherical nanoparticle (yellow beads) is placed above the gel layer and fibers, so that the particle does not interact with either the gel or the fiber beads. The entire system is immersed in a host solution.

To model the dynamic behavior of this system, we utilize dissipative particle dynamics (DPD), ${ }^{6-8}$ which is a particle-based approach used to simulate the time evolution of a many-body system governed by Newton's equation of motion, $m d \mathbf{v}_{i} / d t=\mathbf{f}_{i}$. Each bead $i$ in the system experiences a force $\mathbf{f}_{i}$ that is the sum of three pairwise additive forces: $\mathbf{f}_{i}(t)=\sum\left(\mathbf{F}_{i j}^{\mathrm{C}}+\mathbf{F}_{i j}^{\mathrm{D}}+\mathbf{F}_{i j}^{\mathrm{R}}\right)$, where the sum is over all beads $j$ within a certain cutoff radius $r_{\mathrm{c}}$ from bead $i$. The three forces 
are the conservative force $\mathbf{F}_{i j}^{\mathrm{C}}$, drag or dissipative force $\mathbf{F}_{i j}^{\mathrm{D}}$, and random force $\mathbf{F}_{i j}^{\mathrm{R}}$. We describe each pairwise force below.

The conservative force is a soft, repulsive force given by $\mathbf{F}_{i j}^{\mathrm{C}}=a_{i j}\left(1-r_{i j}\right) \hat{\mathbf{r}}_{i j}$, where $a_{i j}$ measures the maximum repulsion between beads $i$ and $j, r_{i j}=\left|\mathbf{r}_{i}-\mathbf{r}_{j}\right| / r_{\mathrm{c}}$, and $\hat{\mathbf{r}}_{i j}=\left(\mathbf{r}_{i}-\mathbf{r}_{j}\right) /\left|\mathbf{r}_{i}-\mathbf{r}_{j}\right|$. This soft-core force leads to a degree of overlap between neighboring beads and permits the use of larger time steps than those typically used in MD simulations, ${ }^{8}$ which commonly involve the use of hard-core potentials (e.g., the Lennard-Jones potential). The interaction parameter $a_{i j}$ is given in terms of $k_{B} T / r_{c}$, where $r_{c}$ is the characteristic length scale in our simulations. We choose room temperature as the reference value and the corresponding energy scale $k_{B} T_{0}$ with $T_{0}=25^{\circ} \mathrm{C}$ as the characteristic energy scale. Thus, we set $k_{B} T=1$ at $T=25^{\circ} \mathrm{C}$. The reduced temperature is then introduced as $T^{*}=\frac{T}{T_{0}}$, and hence, $T^{*}=1.077$ for $T=48^{\circ} \mathrm{C}$ and $T^{*}=1.01$ for $T=28^{\circ} \mathrm{C}$. All the interaction parameters are presented as functions of the reduced temperature $T^{*}$ (see Table 1).

The drag force is $\mathbf{F}_{i j}^{\mathrm{D}}=-\gamma \omega_{\mathrm{D}}\left(r_{i j}\right)\left(\hat{\mathbf{r}}_{i j} \cdot \mathbf{v}_{i j}\right) \hat{\mathbf{r}}_{i j}$, where $\gamma$ is a simulation parameter related to viscosity arising from the interactions between beads, $\omega_{\mathrm{D}}$ is a weight function that goes to zero at $r_{\mathrm{c}}$, and $\mathbf{v}_{i j}=\mathbf{v}_{i}-\mathbf{v}_{j}$. The random force is $\mathbf{F}_{i j}^{\mathrm{R}}=\sigma \omega_{\mathrm{R}}\left(r_{i j}\right) \xi_{i j} \hat{\mathbf{r}}_{i j}$, where $\xi_{i j}$ is a zero-mean Gaussian random variable of unit variance and $\sigma^{2}=2 k_{\mathrm{B}} T \gamma$ relates the amplitude of the noise to the friction coefficient, as specified by the fluctuation-dissipation theorem. ${ }^{7,8}$ The value of $\gamma=4.5$ is chosen to ensure relatively rapid equilibration of the temperature of the system and the numerical stability of the simulations for the specified time step. ${ }^{8}$ Finally, we use 
$\omega_{\mathrm{D}}\left(r_{i j}\right)=\omega_{\mathrm{R}}\left(r_{i j}\right)^{2}=\left(1-r_{i j}\right)^{2}$ for $r_{i j}<1 .^{8}$

Each of these three pairwise forces conserves momentum locally, and thus, DPD reproduces correct hydrodynamic behavior. $^{6-8}$ The velocity-Verlet algorithm is applied to integrate the equations of motion in time. We take $r_{\mathrm{c}}$ as the characteristic length scale and $k_{\mathrm{B}} T$ as the characteristic energy scale in our simulations. The corresponding characteristic time scale is then defined as $\tau_{\text {intrinsic }}=\sqrt{m r_{\mathrm{c}}^{2} / k_{\mathrm{B}} T}$. The remaining simulation parameters are $\sigma=3$ and $\Delta t=0.02 \tau$, with a total bead number density of $\rho=3 .^{8}$

We take the initial configuration of the gel to be a finite-sized tetra-functional network with a diamond-like topology. ${ }^{9,10}$ The semi-flexible polymer strands are modeled as a sequence of 30 DPD beads that are connected by harmonic bonds, with an interaction potential given by $E=\frac{1}{2} K_{\text {bond }}\left(r-r_{0}\right)^{2}+K_{\text {angle }}(1+\cos \theta) .{ }^{11,12}$ The first term in the latter expression characterizes the elastic energy with the elastic constant $K_{\text {bond }}$ and the second term represents the bending energy with the rigidity parameter $K_{\text {angle }}$. Here, $r_{0}$ is the equilibrium bond length and $\theta$ is the bond angle between two adjacent bonds. The bond and angle potentials of the gel are set respectively at $K_{\text {bond }}=128$ and $K_{\text {angle }}=4$ to prevent bond crossing, and produce a polymer concentration comparable to the experimental results for this gel. ${ }^{13,14}$ Consequently, the total force acting on each geal bead is equal to $\mathbf{f}_{e}+\mathbf{f}_{i}$, where $\mathbf{f}_{e}=-\nabla E$ and $\mathbf{f}_{i}$ is the DPD pairwise force.

As in our previous studies, ${ }^{10}$ the finite-sized network contains cross-links, which are beads with a connectivity of four, and dangling ends, which are located on the surface of the lattice and have a connectivity of less than four. Here, the gel contains 12096 beads and consists 
of 396 strands, 175 cross-links, and 41 dangling ends. This network is periodic in the lateral $(x$ and $y$ ) directions.

To properly model the thermo-responsive behavior of this gel, we relate the repulsive parameter between a polymer and solvent bead, $a_{\mathrm{ps}}$, to the Flory-Huggins parameter characterizing the polymer-solvent interaction, $\chi_{\mathrm{ps}}$, as follows: $a_{\mathrm{ps}}=a+k_{B} T \chi_{\mathrm{ps}} / 0.306 .{ }^{8}$ In studies of thermo-responsive gels (e.g., poly( $N$-isopropylacrylamide) (PNIPAAm)), it is typically assumed that $\chi_{\mathrm{ps}}$ depends on temperature and polymer concentration. ${ }^{15-18}$ Hence, we assume that $\chi_{\mathrm{ps}}(T, \varphi)=\chi_{1}(T)+\chi_{2} \varphi_{\mathrm{P}},{ }^{10}$ where $\varphi_{\mathrm{P}}$ is the polymer volume fraction in the gel and $\chi_{1}(T)=(\delta h-T \delta s) / k_{B} T$, with $\delta h$ and $\delta s$ being the respective changes in enthalpy and entropy. ${ }^{15,19}$ Note that $\varphi_{\mathrm{P}}$ is calculated as $\varphi_{\mathrm{P}}=\bar{\rho}_{\mathrm{P}} / \bar{\rho}_{\text {gel }}$ where $\bar{\rho}_{\mathrm{P}}$ is the time-averaged polymer number density and $\bar{\rho}_{\text {gel }}$ is the time-averaged total number density of the gel including the polymer and solvent beads. Here, we set $a=25$ and take $\delta h=-14.331 \times 10^{-14}$ erg , $\delta s=-5.452 \times 10^{-16} \mathrm{erg} \cdot \mathrm{K}^{-1}$ and $\chi_{2}=0.596$ to produce a continuous volume transition between $T=30^{\circ} \mathrm{C}$ and $35^{\circ} \mathrm{C} .8,10,20-24$ In particular, with this choice of $a_{\mathrm{ps}}$, we reproduce the experimentally observed temperature-induced volume phase transitions of the PNIPAAm gels. ${ }^{10}$ 15,25

The gel layer is attached via an adhesive interaction to the substrate (brown beads in Fig. 1a). The effective attraction between the gel and substrate is modeled by setting the interaction parameter between the beads in the gel and bottom wall, $a_{\mathrm{gw}}$, at $a_{\mathrm{gw}}=a_{\mathrm{ps}}-8$ so that the gel remains anchored to the wall in all our simulations.

The non-deformable spherical particle in Fig. $1 \mathrm{~b}$ is constructed from 1624 DPD beads 
that are dispersed on two spherical layers with an outer layer radius of $R_{\mathrm{p}}=5$ and interlayer spacing 0.5 . Each spherical layer is modeled by geodesic grids generated by subdividing an icosahedron (Fig. 1b), ${ }^{26,27}$ and thus, the vertices of the grids are evenly distributed. In this way, we construct a particle with a well-defined smooth surface, maintaining a high degree of symmetry, with an outer diameter of 10 . The total force and torque acting on this particle is computed as the sum of the forces and torques on its constituent DPD beads. The corresponding number density of the particle shell is 11.4 , which is sufficiently high to prevent penetration of the polymer beads into the sphere and does not induce an unrealistic depletion force between the solvent beads (a behavior that can occur in particle-based simulation methods such as DPD). ${ }^{28}$

The fibers are modeled as semi-flexible polymer chains with a length of 28 units. The $4 \times 4$ array of fibers is anchored onto the substrate with an inter-fiber spacing of 6.15 , as shown in Fig. 1c. The interaction potential for the fibers has the same form as that for the polymer gel: $E=\frac{1}{2} K_{\text {bond }}^{f}\left(r-r_{0}\right)^{2}+K_{\text {angle }}^{f}(1+\cos \theta)$. In these studies, we fix $K_{\text {bond }}^{f}=200$ and vary the rigidity parameter $K_{\text {angle }}^{f}$ from $10^{3}$ to $2 \times 10^{4}$. We also consider the following values for the particle-fiber interaction parameter: $a_{\mathrm{pf}}=20,25$ and 30 .

The top and bottom solid walls that bound the system in the $z$ direction are modeled as solid beads (brown beads in Fig. 1a) with a height $h=1$ and density $\rho_{\text {wall }}=3$. (The wall beads are organized in an amorphous arrangement.) Bounce-back boundary conditions are applied at the fluid-solid interfaces to prevent the solvent and gel beads from penetrating into the walls, and to produce no-slip boundary conditions with minimal interfacial density oscillations. ${ }^{12}$ Periodic boundary conditions are applied along the $x$ and $y$ directions. 
Shear is applied to the system by moving the upper wall of the simulation box along the $x$ direction at specified velocities. The shear rate $\dot{\gamma}$ takes the values $1.67 \times 10^{-4}$ and $1.67 \times 10^{-2}$, which correspond respectively to the velocities of the upper wall of 0.01 and 1 in dimensionless units.

Finally, the simulation box is $24.6 \times 24.6 \times 60$ units in size and is filled with 89603 solvent beads, maintaining the total density of the system at $\rho_{\text {sys }}=3$. Eight independent simulations are carried out for $t=5 \times 10^{6}$ time steps for each parameter set. (In the ensuing discussion, $t$ is specified in simulation time steps, which can be related to physical units of time as discussed below.)

\section{B. Comparison of simulation parameters to physical values}

We can relate the dimensionless parameters to physical values through the value of the collective diffusion coefficient of the polymer network. If we assume that each solvent bead represents 10 water molecules, ${ }^{29,30}$ then a DPD solvent bead occupies a volume of $300 \mathrm{~A}^{3}$ since a water molecule (of mass density $1 \mathrm{~g} / \mathrm{cm}^{3}$ ), has a volume $\sim 30 \AA^{3}$. The total bead number density in our system is $\rho_{\text {sys }}=3$ and using $\rho_{\text {sys }}=3 r_{c}^{-3}$ and the mass density of water, we obtain the unit length $r_{c}=0.97 \mathrm{~nm}$ and the characteristic mass $m=180 \mathrm{Da}$. By matching the mass density of a polymer bead in the simulation to the mass density of amorphous PNIPAAm $\left(1.1 \mathrm{~g} / \mathrm{cm}^{3}\right)$, we find that a polymer bead represents 1.6 PNIPAAm monomers. ${ }^{10}$

Knowing the characteristic length, mass and energy, the time scale for the DPD simulation is calculated as $\tau_{\text {intrinsic }}=\sqrt{m r_{c}^{2} / k_{B} T_{0}}=8.3 \mathrm{ps}$. It is known that conventional DPD produces accelerated dynamics due to the soft-core potential if the above intrinsic time scale is 
used. $^{8,31,32}$ To obtain the correct characteristic time scale, we relate the collective diffusion coefficient of the polymer network in the simulations, $\mathrm{D}_{0}^{\mathrm{sim}}=1.74 \times 10^{-2} \mathrm{~nm}^{2} / \tau_{\mathrm{DPD}}$, obtained from the swelling kinetics of the gel, to the experimental value $D_{0}^{\exp }=2 \times 10^{-11} \mathrm{~m}^{2} / \mathrm{s} .{ }^{33}$ We thus obtain the following physical values for the simulation parameters: $\tau_{\mathrm{DPD}}=0.87 \mathrm{~ns}$, the simulation box size is $23.9 \times 23.9 \times 58.2 \mathrm{~nm}$, the fiber equilibrium length is $27.2 \mathrm{~nm}$, and the particle diameter is $9.7 \mathrm{~nm}$. The maximum value of the applied shear rate $1.67 \times 10^{-2}$ corresponds to $\dot{\gamma}=1.9 \times 10^{7} / \mathrm{s}^{34,35} K_{\text {bond }}=128$ and $K_{\text {angle }}=4$ correspond to $0.56 \mathrm{~N} / \mathrm{mand} 4 \mathrm{k}_{b} T$, respectively.

To relate the parameter that characterizes the stiffness of the fiber in the simulation, $K_{\text {angle }}^{f}$, to physical values, we calculate the persistence length as: $l_{p}=2 r_{0} \frac{\left(N_{f}-2\right)}{\left(N_{f}-1\right)} \frac{K_{\text {angle }}^{f}}{k_{b} T}$, where $r_{0}=0.5$ is the equilibrium bond length of the fiber and $N_{f}=28$ is the number of beads in the

fiber. ${ }^{12,36}$ The unit of length in our simulations is $0.97 \mathrm{~nm}$. So that $K_{\text {angle }}^{f}=1,000$ corresponds to $0.96 \mu \mathrm{m}$ and $K_{\text {angle }}^{f}=20,000$ corresponds to $19.3 \mu \mathrm{m}$. The latter values correspond, for example, to the persistence lengths of bio-filaments, which can range from $0.2 \sim 20 \mu \mathrm{m} .{ }^{37}$

\section{Results and Discussions}

At the outset of the simulations, the polymer gel is equilibrated at the temperature $T=28^{\circ} \mathrm{C}$ and the nanoparticle is located at $Z_{c}=37$, where $Z_{c}$ is the vertical position of the bottom of the particle, as shown in Fig. 2a. An imposed shear drives the fluid to flow from left to right along the $x$ direction; the shear rate is set at the lowest value considered here, $\dot{\gamma}=1.67 \times 10^{-4}$. Recall that the gel is attached to the bottom wall, which has a height of 1 . We denote the center of mass of the gel as $\left\langle Z_{\text {gel }}\right\rangle$, which is obtained by averaging over the $\mathrm{z}$ 
coordinates of all the gel beads. (Given the coordinate vector of the $i$ th gel bead, $\mathbf{r}_{i}$, the total number of beads $N$, and the mass of the bead being equal to 1 , the center of mass is $\sum_{i} \mathbf{r}_{i} / N$; projecting the center of mass vector onto the $z$ direction, we get $Z_{g e l}=\sum_{i} z_{i} / N$.) Hence, the height of the gel/fluid interface, $h_{\text {gel }}$, is approximated as $h_{\text {gel }}=2\left(\left\langle Z_{\text {gel }}\right\rangle-1\right)+1=2\left\langle Z_{\text {gel }}\right\rangle-1$. This calculation yields the equilibrium value of $h_{\mathrm{gel}} \approx 30$ at $T=28^{\circ} \mathrm{C}$. The flexible fibers are 28 units in length and are initially aligned with the $z$-axis (Fig. 1a). Hence, at the beginning of the simulations, the nanoparticle does not come into contact with the polymer gel or the fibers. Periodic boundary conditions are applied along the lateral direction of the simulation box.

The entire system (under the imposed shear) is relaxed for $5 \times 10^{3}$ time steps before the temperature of the system is increased to $T=48^{\circ} \mathrm{C}$. The gel displays a LCST, and thus, collapses with an increase in $T$ due to the temperature-dependent increase in the gel-solvent interaction parameter. ${ }^{10}$ Hence, at $T=48^{\circ} \mathrm{C}$, the gel height decreases to a new equilibrium value of $h_{\text {gel }} \approx 10.8$ because of the now unfavorable enthalpic interaction with the solution. As noted in the Introduction, the shrinking of the gel does not cause the relatively stiff fibers to collapse. As detailed in the SI, for $K_{\text {angle }}^{f}=10^{3}$, the fibers extend through the gel as the height of the gel decreases.

Both the fibers and the particle are hydrophobic (see Table 1) and equally incompatible with the gel. Notably, when the gel is swollen (at $T=28^{\circ} \mathrm{C}$ ), the excluded volume interaction between the fiber and the surrounding gel prevents the embedded fibers from completely "bunching" together to avoid the unfavorable enthalpic interactions. (Without the gel, the fibers in close proximity will aggregate into bundles to minimize contact with the incompatible fluid; 
the penalty associated with the stretching of the neighboring chains is outweighed by the gain in enthalpy.)

As the gel collapses at $T=48^{\circ} \mathrm{C}$, however, the exposed fibers are less sterically constrained by the gel, and thus, can more readily interact not only with each other, but also with the nanoparticle (Figs. $2 \mathrm{~b}$ and $2 \mathrm{c}$ ). The nanoparticle exhibits a favorable interaction with the fibers; moreover, the relative particle-fiber attraction is stronger than the fiber-fiber attraction. Hence, if the particle is convected sufficiently close to the fibers (i.e., within the cutoff distance of $r_{c}$ ), then the fibers will preferentially bind to the surface of the particle. Thus, the interplay among the thermo-responsive gel, the exposed fibers, and the adhesive particle provides an effective approach for extracting the nanoparticles from the solution. Moreover, the particles are inhibited from re-entering the solution by the encircling fibers (Fig. 2c).

For $K_{\text {angle }}^{f}=10^{3}$, the stiff fibers are still relatively flexible; a more detailed examination of Fig. 2 provides additional insight into the behavior of this system. In particular, once these hydrophobic fibers become exposed (Fig. 2b), they stretch toward each other and self-assemble into bundles (thereby replacing unfavorable fiber-solvent contacts with favorable fiber-fiber contacts). When the adhesive particle in the flowing fluid comes within the interaction range of the fibers, these fibers are sufficiently compliant that they can wrap around the nanoparticle, thereby increasing the number of fiber-particle contacts. In this case, the increase in the elastic energy of the fibers due to stretching is offset by the favorable particle-fiber enthalpic interactions. The adhesive interaction continues to increase as more contacts are formed between the fibers and nanoparticle (Fig. 3); this facilitates the extraction process and the delivery of the nanoparticle into the gel. As shown in Fig. 2b, at $t=5 \times 10^{5}$, a multi-fiber bundle has effectively trapped the nanoparticle, which is partially immersed in the polymer gel; as seen in Fig. 2c, the 
particle becomes completely immersed in the gel layer at $t=5 \times 10^{6}$. Notably, fibers that do not interact with the nanoparticle are still available for extracting other particles.

To quantify the process of extracting the nanoparticle from solution, we monitor the temporal evolution of $Z_{c}$ (the vertical position of the bottom of the particle) and $h_{\text {gel }}$ (the height of the gel), as shown by the black curves in Fig. $3 a$ and $3 b$, respectively. The shading about the lines represents the corresponding variations among eight independent runs. Figure $3 a$ shows that $Z_{c}$ decreases rapidly with time and after a short period $\left(t \approx 10^{4}\right)$, its value is less than 28 , which is the height of the initially extended fiber; this suggests that the fibers and particle come into contact at relatively early times. In other words, the fibers are relatively efficient in detecting and catching the nanoparticle. Thereafter, $Z_{c}$ continues a slow decrease until $t \approx 2 \times 10^{6}$, becoming less than $h_{\text {gel }}$. As the thermo-responsive gel collapses, the number density of gel beads increases, and thus, generates a greater exclusion force on the nanoparticle. Consequently, the curve reaches a plateau and at $t=5 \times 10^{6}$, attaining a constant value of $Z_{c} \approx 2.1$. Recall that the substrate is composed of DPD beads of height 1.0 and the range of repulsion between the bottom wall and the nanoparticle is also 1.0. Hence, the latter value of $Z_{c}$ indicates that the nanoparticle reaches the bottom wall of the simulation box.

Figure $3 \mathrm{~b}$ reveals not only the collapse of the LCST gel in response to the increase in temperature, but also readjustments of the layer due to the presence of the nanoparticle. In particular, it is notable that at $t=5 \times 10^{6}$, the gel height is $h_{\text {gel }} \approx 13.4$; this value is greater than the equilibrium value of 10.8. This increase in $h_{\text {gel }}$, together with the image in Fig. 2c, indicates that the nanoparticle is completely immersed into the polymer gel. Namely, the particle takes up volume previously occupied by the gel, which is now pushed to lie above the inclusion. 
We anticipate that the ability of the fibers to trap the nanoparticle will depend on the relative flexibility of the fiber. To test this hypothesis, we investigate the effect of varying $K_{\text {angle }}^{f}$, and thus, the rigidity of the fiber, on the performance of the system. Notably, as the bending rigidity of the fiber is increased to $K_{\text {angle }}^{f}=10^{4}$, the temporal evolution of $Z_{c}$ is distinctly different from that for the more flexible chains, as can be seen by examining the red curve in Fig 3a. Clearly, it takes a relatively longer time for the more rigid fibers to draw the nanoparticle toward the lower wall than in the previous case. As illustrated in Fig. $4 \mathrm{a}$, at $t \approx 10^{5}$, the particle still lies near the top of the gel and has few contacts with the fibers. This behavior is due to two factors. First, the rigid fibers cannot wrap around the particle as readily as the more compliant ones. Second, the stiffer fibers do not self-assemble into bundles. In both cases, the energetic cost of bending is not compensated by the energetic gains. Both these factors lead to fewer points of contact between the fibers and particles, as can be seen from the evolution of $N_{\text {pf }}$ (Fig. $3 \mathrm{c}$ ), the number of contacts between the particle and the fiber beads. (The different beads are considered to be in contact if they are separated by a distance that is less than or equal to $r_{c}$, the interaction range.) Thus, fibers with higher rigidity are less effective at pulling the particle into the gel layer. As shown in Fig. 4b and 4c, even though the particle is extracted from the outer fluid, it is not transported into the bulk of the gel layer. Rather, the particle remains localized near the top of the gel. At $t=5 \times 10^{6}$ (Fig. $4 \mathrm{c}$ ), $Z_{c} \approx 6.8$ and the average height of the entire gel is equal to the unperturbed equilibrium value of $h_{\mathrm{gel}} \approx 10.8$.

For the most rigid fibers considered here, we set $K_{\text {angle }}^{f}=2 \times 10^{4}$; the data for $Z_{c}$ and $h_{\text {gel }}$ is plotted in green in Figs. $3 \mathrm{a}$ and $3 \mathrm{~b}$, respectively. We can obtain further insight into the behavior of this system from the snapshots in Fig. 5. Figure 5a shows that similar to the case 
with $K_{\text {angle }}^{f}=10^{4}$, the rigid hydrophobic fibers do not self-organize into bundles; this image displays the configuration of the system at $t=10^{5}$, just when the particle and fibers come into contact. As the gel shrinks, the nanoparticle remains arrested on top of the rigid fibers (Fig. 5b) and hence, the conformation of the gel is not affected, consistent with the evolution of $h_{\text {gel }}$. Since there are only a few points of particle-fiber contact (see green curve in Fig. 3c), the fluid-driven nanoparticle can move along the rigid fibers, leading to the large fluctuations in the evolution of $Z_{c}$ seen in Fig. 3a.

Finally, we note that $h_{\text {gel }}$ in the case of the stiffer fibers (see red and green curves in Fig. $3 \mathrm{~b}$ and black curve in Fig. 6) exhibits a pronounced decrease around $t=2 \times 10^{6}$ relative to the scenario involving the compliant fibers. With the particle being localized relatively high in the layer (see Fig. 3a), the space near the substrate becomes filled by the gel beads; this leads to the decrease in the height of the gel.

The value of interaction parameter between the particle and fiber will also affect the ability of the system to extract the particle from the solution. We decreased the strength of the adhesion between these components by setting $a_{\mathrm{pf}}=25$ and 30 (see Table 1) and examined the behavior of the system for the most flexible and rigid fibers considered here, i.e., $K_{\text {angle }}^{f}=10^{3}$ and $2 \times 10^{4}$. For each of these $K_{\text {angle }}^{f}$ values, the decrease in the adhesive strength produced quantitatively similar results to the respective case with $a_{\mathrm{pf}}=20$. In other words, this increase in $a_{\mathrm{pf}}$ had a less dramatic effect on the system than altering the value of $K_{\text {angle }}^{f}$. This finding can be understood by examining the number of contacts, $N_{\mathrm{pf}}$, between the fiber and particle beads. For $K_{\text {angle }}^{f}=10^{3}, N_{\mathrm{pf}} \approx 175$, while for $K_{\text {angle }}^{f}=2 \times 10^{4}, N_{\mathrm{pf}} \approx 10$ for the reference 
case of $a_{\mathrm{pf}}=20$ (see Fig. 3c). Recall all forces in DPD are repulsive and the particle-solvent interaction is 40 (Table 1). Thus, the effective adhesive force acting on the nanoparticle due to the fiber is approximately proportional to $N_{\mathrm{pf}}\left(40-a_{\mathrm{pf}}\right)$. (We note that the simulation results reveal that $N_{\mathrm{pf}}$ is insensitive to $a_{\mathrm{pf}}$ for the range of $a_{\mathrm{pf}}$ considered here.) Given that the values of $N_{\mathrm{pf}}$ differ by an order of magnitude for these two extreme values of $K_{\text {angle }}^{f}$, small changes in $a_{\mathrm{pf}}$ will not affect the respective behavior of these systems. Hence, the effectiveness of the flexible "grippers" illustrated in Fig. 2c is not reduced by such changes in the adhesiveness of the particle. This finding implies that this extraction mechanism could be applied to nanoparticles with a variety of surface chemistries.

Notably, however, the rate of the imposed shear could affect the performance of the system. To test this hypothesis, we focus on the limit of relatively high shear and set the shear rate to $\dot{\gamma}=1.67 \times 10^{-2}$. Keeping $a_{\mathrm{pf}}=20$, we examine the behavior of system for both $K_{\text {angle }}^{f}=10^{3}$ and $2 \times 10^{4}$. For the most flexible fibers $\left(K_{\text {angle }}^{f}=10^{3}\right)$, we observe that in six of the eight independent simulations, the fibers catch and bind the fluid-driven nanoparticles, as illustrated in Figs. $6 \mathrm{c}$ and $6 \mathrm{~d}$. In the other two cases, the particles were initially caught by the fibers, but the drag force from the fluid was sufficiently strong to break this binding and the nanoparticle was released to the solution.

For the six cases where the particle was successfully caught, we analyze the temporal evolution of $Z_{c}$ (red curve) and $h_{\text {gel }}$ (black curve), as shown in Fig. 6a. As the gel collapses, $Z_{c}$ first fluctuates for about $1.15 \times 10^{6}$ time steps before decreasing to a value less than $h_{\text {gel }}$. Comparison with Figs. $3 \mathrm{a}$ and $3 \mathrm{~b}$ indicates that at a high shear rate, the fibers take a much longer time to detect the presence of the nanoparticle than at the lower value of $\dot{\gamma}$. The large error bars 
around $Z_{c}$ indicate that the position of the particle undergoes large fluctuations since the fluid strongly disturbs the motion of the nanoparticle.

Ultimately, the fibers successfully catch and wrap around the nanoparticle at $t \approx 1.15 \times 10^{6}$, as shown in Fig. 6a. Notice that the fibers bend along the direction of shear due to the strong drag force. For $t \geq 4.1 \times 10^{6}$, the significant fluctuations vanish since at this late stage, the fibers completely surround the nanoparticle and the adhesion overcomes the shear force. As shown in Fig. 6d, the nanoparticle is arrested by the fibers and the points of contact between the particle and fibers appear to have reached a maximal value. The gel interface $h_{\text {gel }}$ is not significantly affected by the shear and that fact that $Z_{c}<h_{\text {gel }}$ indicates that the nanoparticle is trapped within the gel layer.

For the most rigid fibers ( $K_{\text {angle }}^{f}=2 \times 10^{4}$ ), no catch is observed in the eight independent simulations. As indicated in Fig. $6 b, Z_{c}$ only fluctuates around 40, which is close to the initial input value of 37 . As discussed above, the number of contacts between the nanoparticle and the fibers is less than that for $K_{\text {angle }}^{f}=10^{3}$ by an order of magnitude. The resulting small adhesive interaction cannot hold the nanoparticle for long times against the effects of the imposed flow at this high shear rate.

The above findings suggest that the nanoparticle can be held at a distinct height within the gel layer and could be released "on demand" by an imposed shear. To illustrate this point, we take the final systems (at $t=5 \times 10^{6}$ ) obtained in each one of the previous eight simulation runs for each of the parameter sets as given in Fig. 3 as the initial configurations, and apply high shear $\dot{\gamma}=1.67 \times 10^{-2}$ to the arrested nanoparticle for $5 \times 10^{6}$ time steps. For the most flexible fibers 
( $K_{\text {angle }}^{f}=10^{3}$ ), the nanoparticle that is initially wrapped by the fibers remains embedded in the gel due to the strong fiber-particle adhesion in all eight simulation runs.

For the intermediate value of the fiber rigidity $\left(K_{\text {angle }}^{f}=10^{4}\right)$, we find that in six of the eight simulations, the nanoparticle that is located near the top of the gel is dislodged from the fibers. As shown in Fig. 7a, at $t=2.5 \times 10^{5}$, the shear drives the particle to start detaching from the gel layer. As the particle is uplifted by the flow at $t=5 \times 10^{5}$ (Fig. 7b), the number of particlefiber contact points decreases, which in turn reduces the adhesive strength between the particle and the fibers. At the late stage at $t=7.5 \times 10^{5}$ (Fig. 7c), the nanoparticle is released from the fibers.

Finally, in the case of the most rigid fibers $\left(K_{\text {angle }}^{f}=2 \times 10^{4}\right)$, the shear force is sufficiently large to overcome the adh esion and leads to significantly faster (on average within approximately $1.6 \times 10^{5}$ time steps) release of the nanoparticle from the fibers in all eight simulations

These simulations demonstrate that by tailoring the flexibility of the fibers, one can tune the functionality of the gel-fiber composite. Specifically, the use of flexible fibers results in the robust catching of the nanoparticles and prohibits their release even under high shear flows. On the other hand, more rigid fibers allow one to control both the catching of the nanoparticles and their "on demand" release by applying a shear flow.

\section{Conclusions}

Using DPD simulations, we proposed the design of a gel-fiber coating where the components of the system act in concert to extract particles from solution and localize these solids in the underlying gel layer. The LCST of the gel (representing, for example, PNIPAAm) causes the polymer network to collapse with an increase in temperature and expose the 
hydrophobic fibers. If the fibers are sufficiently flexible, they self-assemble into distinct bundles. When a hydrophobic particle comes sufficiently close to these fibers, the bundles collectively enwrap the particle, thereby shielding it from interactions with the unfavorable solvent. As the number of enthalpically favorable particle-fiber contacts increases with time, the particle is effectively drawn closer to the underlying substrate and hence, deeper into the gel coating. In this manner, the particles are removed from the solution and incorporated into the polymer network.

The efficacy of this mode of particle extraction depends on the flexibility of the fibers, the fiber-particle interaction and the shear rate in the fluid flowing above the gel-fiber coating. In the studies described above, we isolated the optimal parameter range that yielded the robust removal of the particles from the fluid. Hence, we pinpointed the design space where the gelfiber coating could act as an effective filtration system. With this choice of parameters, the system could also provide a means of fabricating gel-nanoparticle composites since the particles become firmly embedded in the gel layer. In particular, even relatively high shear could not dislodge the particle once it was enwrapped by the flexible fibers and entrapped within the gel.

It is also worth noting that this system could be used to load drugs into a polymeric matrix and permit controlled delivery of medication. ${ }^{38}$ In this context, the particles in our simulation represent the drug molecule. For this application, however, it is desirable that the bound particles could be released under a change in environmental conditions. Hence, for the controlled release applications, it is optimal to harness the more rigid fibers, which enable the particles to be localized within the "forest" of fibers, but not be completely trapped. Specifically, the application of shear can dislodge particles from near the tops of the fibers.

The ability to dislodge trapped particles with an increase in the shear rate also makes the systems with rigid fibers applicable for anti-fouling applications. Namely, the latter coating can 
be used to localize the fouling agents near the tips of the fibers. By washing the layer, the particulates can be removed from the surface and the cleaned coating can be used again to trap contaminants, which can be readily removed in a subsequent washing.

\section{Acknowledgements}

ACB and JA are grateful for financial support from DOE DE-SC0005247 for partial support of Olga Kuksenok and the ONR N00014-15-1-2306 for partial support of Ya Liu.

\section{REFERENCES}

1 E. Krol, B. J. Plachno, L. Adamec, M. Stolarz, H. Dziubinska and K. Trebacz, Ann. Bot., 2012, 109, 47.

2 B. Pokroy, S. H. Kang, L. Mahadevan and J. Aizenberg, Science, 2009, 323, 237.

3 A. Shastri, L. M. McGregor, Y. Liu, V. Harris, H. Q. Nan, M. Mujica, Y. Vasquez, A. Bhattacharya, Y. T. Ma, M. Aizenberg, O. Kuksenok, A. C. Balazs, J. Aizenberg and X. M. He, Nat. Chem., 2015, 7, 447.

4 S. Christau, T. Moeller, Z. Yenice, J. Genzer and R. von Klitzing, Langmuir, 2014, 30, 13033.

5 D. Das, T. Kar and P. K. Das, Soft Matter, 2012, 8, 2348.

$6 \quad$ P. J. Hoogerbrugge and J. M. V. A. Koelman, Europhys. Lett., 1992, 19, 155.

$7 \quad$ P. Español and P. Warren, Europhys. Lett., 1995, 30, 191.

8 R. D. Groot and P. B. Warren, J. Chem. Phys., 1997, 107, 4423.

9 P. K. Jha, J. W. Zwanikken, F. A. Detcheverry, J. J. de Pablo and M. Olvera de la Cruz, Soft Matter, 2011, 7, 5965.

10 X. Yong, O. Kuksenok, K. Matyjaszewski and A. C. Balazs, Nano Lett., 2013, 13, 6269. 
11 Y. Liu, G. McFarlin, X. Yong, O. Kuksenok and A.C. Balazs, Langmuir, DOI: 10.1021/acs.langmuir.5b00888.

12 W. Pan, D. Fedosov, G. Karniadakis and B. Caswell, Phys. Rev. E, 2008, 78, 046706.

13 P. Nikunen, I. Vattulainen and M. Karttunen, Phys. Rev. E, 2007, 75, 036713.

14 A. Karatrantos, N. Clarke, R. J. Composto and K. I. Winey, Soft Matter, 2013, 9, 3877.

15 S. Hirotsu, J. Chem. Phys., 1991, 94, 3949.

16 M. Quesada-Pérez, J. A. Maroto-Centeno, J. Forcada and R. Hidalgo-Alvarez, Soft Matter, 2011, 7, 10536.

17 S. Hirotsu, J. Phys. Soc. Jpn., 1987, 56, 233.

18 B. Erman and P. J. Flory, Macromolecules, 1986, 19, 2342.

19 P. J. Flory, Principles of Polymer Chemistry, Cornell University Press, 1953.

20 H.-Y. Chang, Y.-L. Lin, Y.-J. Sheng and H.-K. Tsao, Macromolecules, 2012, 45, 4778.

21 R. D. Groot and T. J. Madden, J. Chem. Phys., 1998, 108, 8713.

22 T. Hellweg, C. D. Dewhurst, Br, x000Fc, E. ckner, K. Kratz and W. Eimer, Colloid Polym. Sci., 2000, 278, 972.

23 J. Guo, H. Liang and Z. G. Wang, J. Chem. Phys., 2011, 134, 244904.

24 C. Soto-Figueroa, M. d. R. Rodríguez-Hidalgo and L. Vicente, Soft Matter, 2012, 8, 1871.

25 H. G. Schild, Prog. Polym. Sci., 1992, 17, 163.

26 S. Chen, N. Phan-Thien, B. C. Khoo and X. J. Fan, Phys. Fluids, 2006, 18, 103605.

27 H. Fan and A. Striolo, Phys. Rev. E, 2012, 86, 051610.

28 I. Salib, X. Yong, E. J. Crabb, N. M. Moellers, G. T. McFarlin, O. Kuksenok and A. C. Balazs, ACS Nano, 2013, 7, 1224. 
29 R. M. Fuchslin, H. Fellermann, A. Eriksson and H. J. Ziock, J. Chem. Phys., 2009, 130, 214102.

30 J. R. Spaeth, I. G. Kevrekidis and A. Z. Panagiotopoulos, J. Chem. Phys., 2011, 134, 164902.

31 V. Symeonidis, G. E. Karniadakis and B. Caswell, J. Chem. Phys., 2006, 125, 184902.

32 C. Junghans, M. Praprotnik and K. Kremer, Soft Matter, 2008, 4, 156.

33 B. Nikoobakht and M. A. El-Sayed, Chem. Mater., 2003, 15, 1957.

34 M. Sega, M. Sbragaglia, L. Biferale and S. Succi, Soft Matter, 2013, 9, 8526.

35 M. Moseler, Science, 2000, 289, 1165.

36 H. Yamakawa, Modern theory of polymer solutions, Harper \& Row, 1971.

37 F. Gittes, B. Mickey, J. Nettleton and J. Howard, J. Cell Biol., 1993, 120, 923.

38 L. C. C. Elliott, B. Jing, B. Akgun, Y. Zhu, P. W. Bohn and S. K. Fullerton-Shirey, Langmuir, 2013, 29, 3259.

\section{Figure Captions}

Figure 1. (a) Initial morphology of the system. (b) Geodesic grid structure of the spherical nanoparticle. (c) Top view of the fiber arrangement.

Figure 2. (a) Initial morphology of the system with the equilibrated swollen gel, fibers and nanoparticle at $T=28^{\circ} \mathrm{C}$ under low shear $\dot{\gamma}=1.67 \times 10^{-4}$. (b-c) Trapping of the particle by the gel composite with the flexible fibers with $K_{\text {angle }}^{f}=10^{3}$. Snapshots of the system taken at $t=5 \times 10^{5}$ for (b) and at $t=5 \times 10^{6}$ for (c).

Figure 3. Images (a)-(c) correspond respectively to the temporal evolution of $Z_{c}, h_{\text {gel }}$, and $N_{\text {pf }}$ for different fiber rigidities: $K_{\text {angle }}^{f}=10^{3}$ (black), $10^{4}$ (red), and $2 \times 10^{4}$ (green).

Figure 4. Trapping of the particle by the gel composite with fibers with intermediate rigidity, $K_{\text {angle }}^{f}=10^{4}$. Images (a)-(c) correspond to $t=10^{5}, 1.5 \times 10^{6}$, and $5 \times 10^{6}$, respectively. 
Figure 5. Particle trapping by the gel composite with fibers with high rigidity, $K_{\text {angle }}^{f}=2 \times 10^{4}$. Images (a)-(c) correspond to snapshots of the system at $t=10^{5}, 2 \times 10^{6}$, and $5 \times 10^{6}$, respectively.

Figure 6. Plots (a) and (b) display the temporal evolution of $Z_{c}$ (red) and $h_{\text {gel }}$ (black) under the shear $\dot{\gamma}=1.67 \times 10^{-2}$ for $K_{\text {angle }}^{f}=10^{3}$ and $2 \times 10^{4}$, respectively. Images (c) and (d) are snapshots of the system for $K_{\text {angle }}^{f}=10^{3}$ at $t=1.15 \times 10^{6}$ and $5 \times 10^{6}$, respectively.

Figure 7. Particle release under the shear $\dot{\gamma}=1.67 \times 10^{-2}$ by the gel composite with the fibers with intermediate rigidity, $K_{\text {angle }}^{f}=10^{4}$. Snapshots at (a)-(c) correspond to $t=0.25 \times 10^{6}$, $0.5 \times 10^{6}$, and $0.75 \times 10^{6}$, respectively.

Table 1

\begin{tabular}{|c|c|c|c|c|c|c|}
\hline & Solvent & Particle & Fiber & $\begin{array}{c}\text { Top } \\
\text { Wall }\end{array}$ & $\begin{array}{c}\text { Bottom } \\
\text { Wall }\end{array}$ & $\begin{array}{c}\text { Polymer } \\
\text { Gel }\end{array}$ \\
\hline Solvent & 25 & 40 & 40 & 25 & 25 & $\chi_{\mathrm{ps}}$ \\
\hline Particle & - & 25 & $a_{\mathrm{pp}}$ & 40 & 40 & 40 \\
\hline Fiber & - & - & 25 & 40 & 40 & 40 \\
\hline Top Wall & - & - & - & 25 & 25 & 25 \\
\hline $\begin{array}{c}\text { Bottom } \\
\text { Wall }\end{array}$ & - & - & - & - & 25 & $a_{\mathrm{gw}}$ \\
\hline $\begin{array}{c}\text { Polymer } \\
\text { Gel }\end{array}$ & - & - & - & - & - & 25 \\
\hline
\end{tabular}


(a)

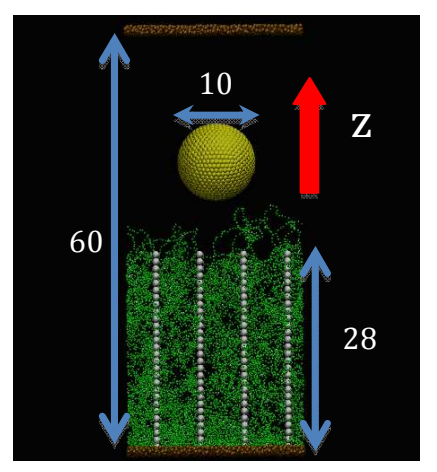

(b)

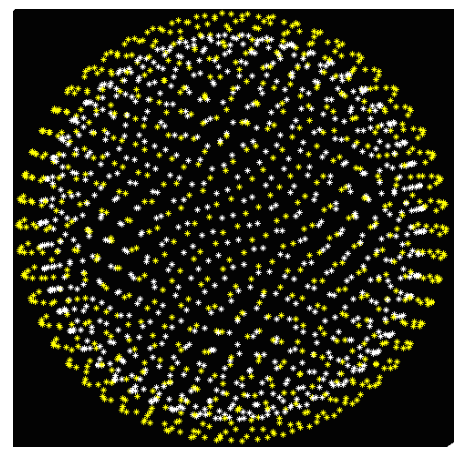

(c)

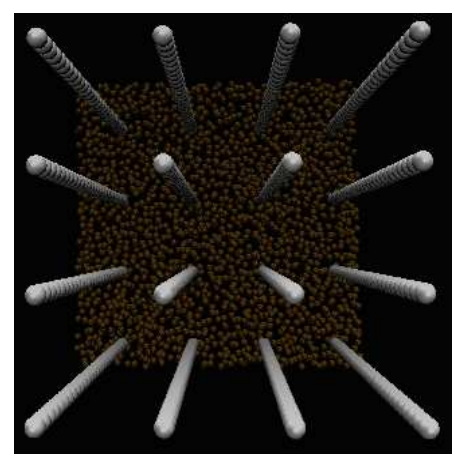

Figure 1 
(a)

(b)

(c)

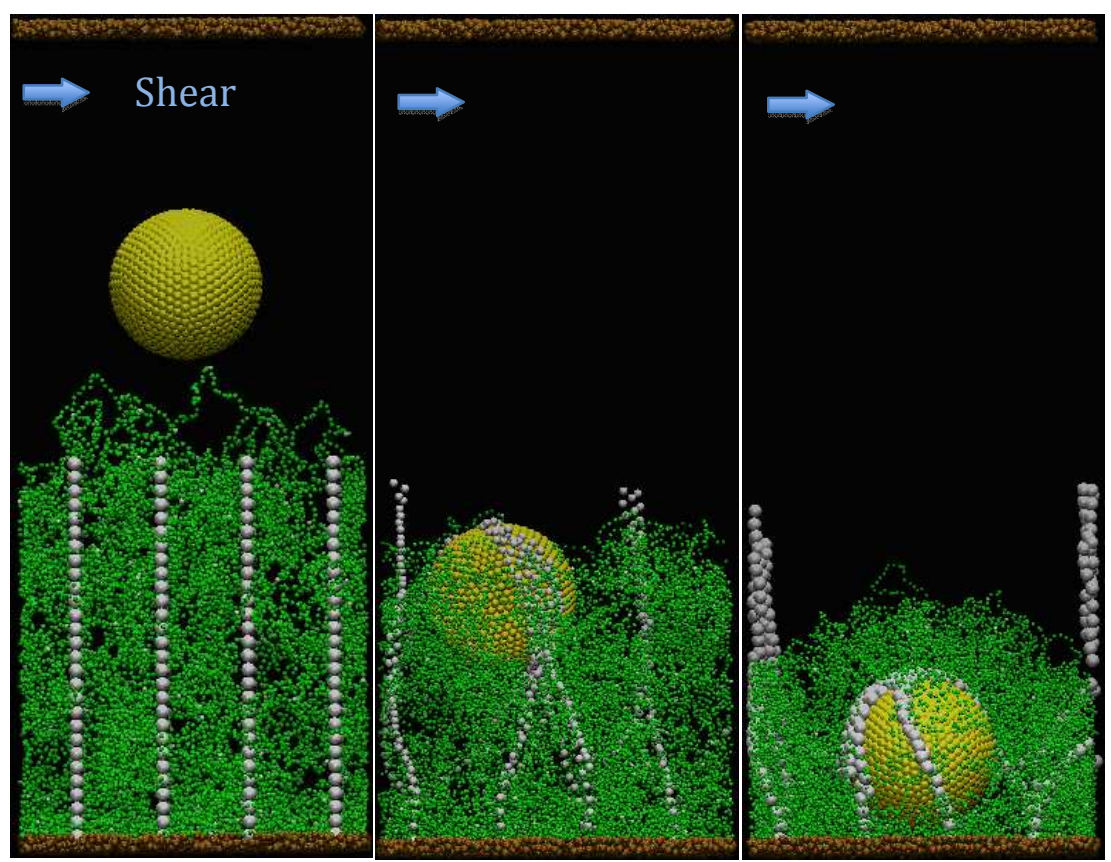

Figure 2 
(a)

(b)
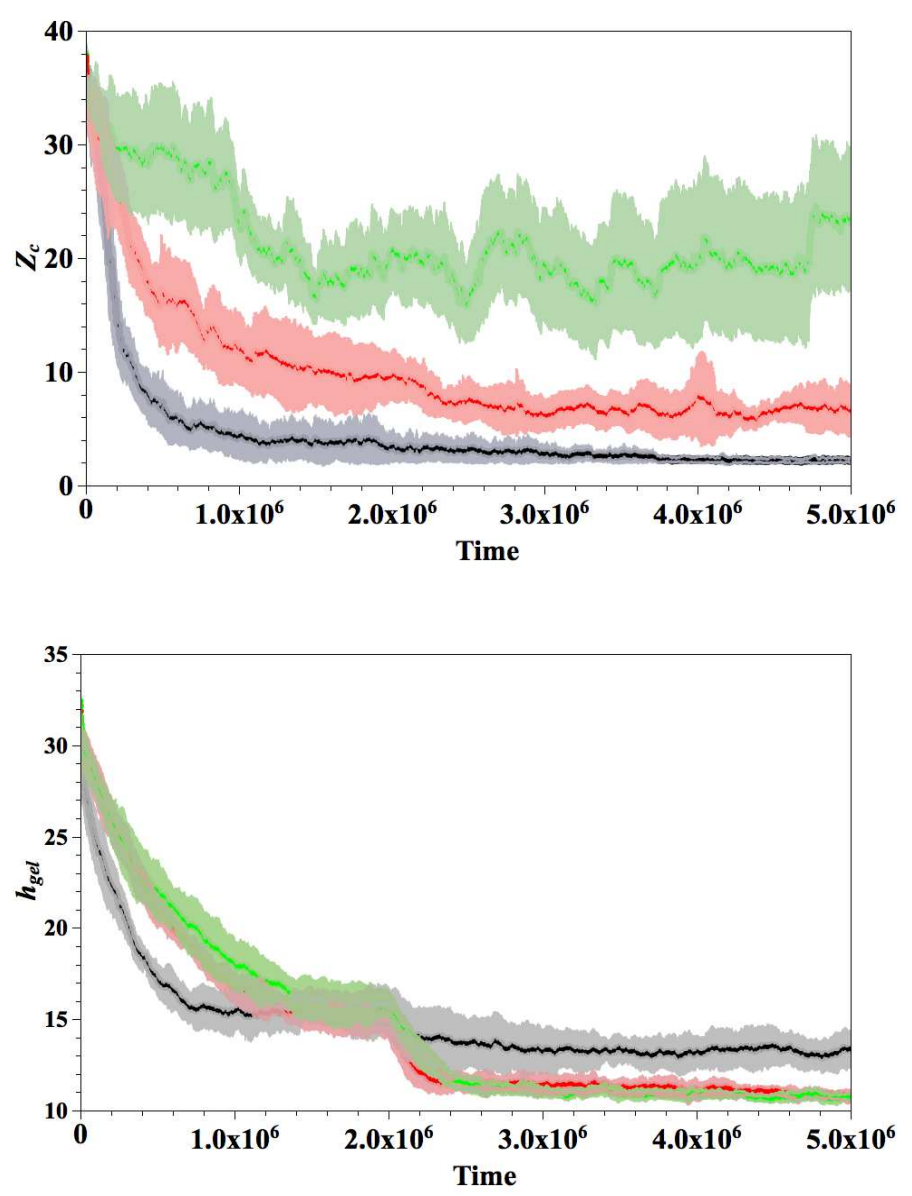

(c)

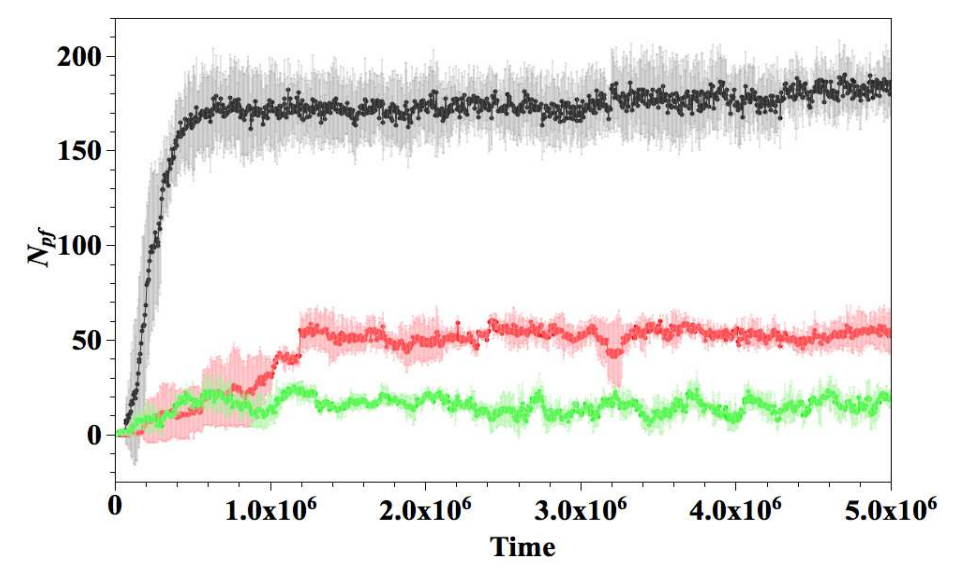

Figure 3 
(a)

(b)

(c)
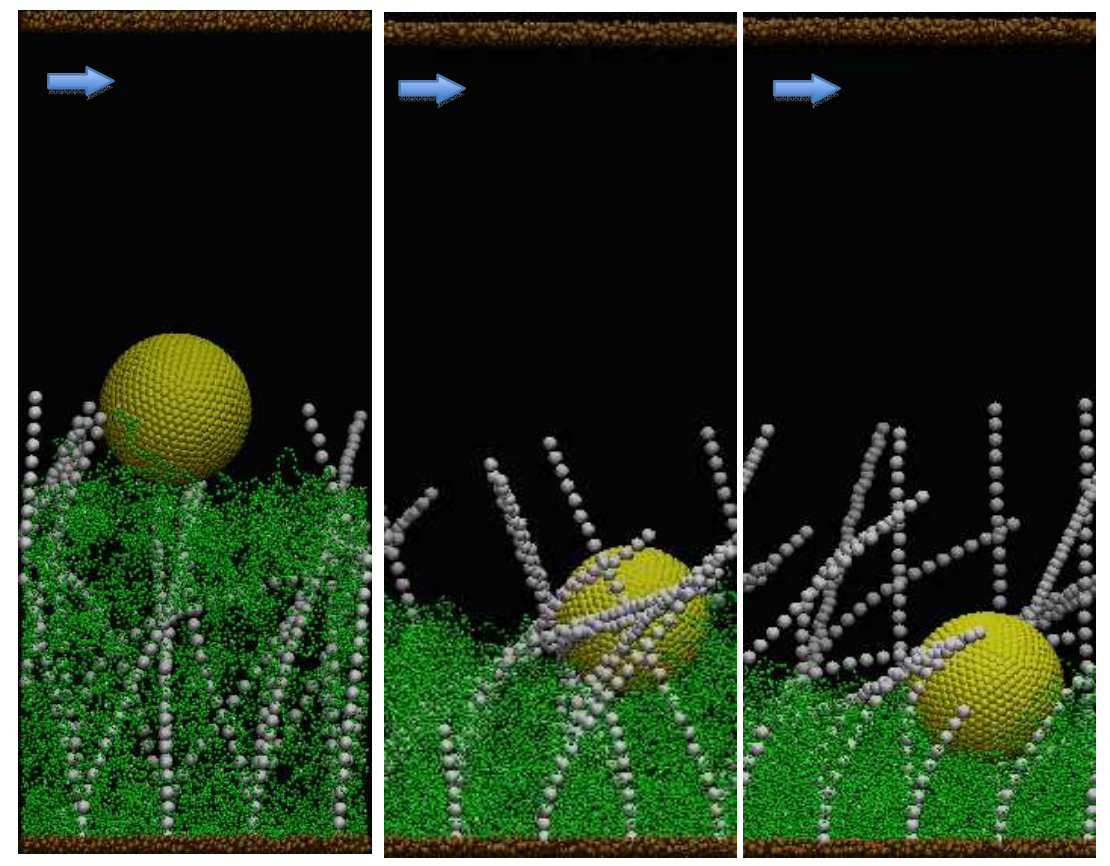

Figure 4 
(a)

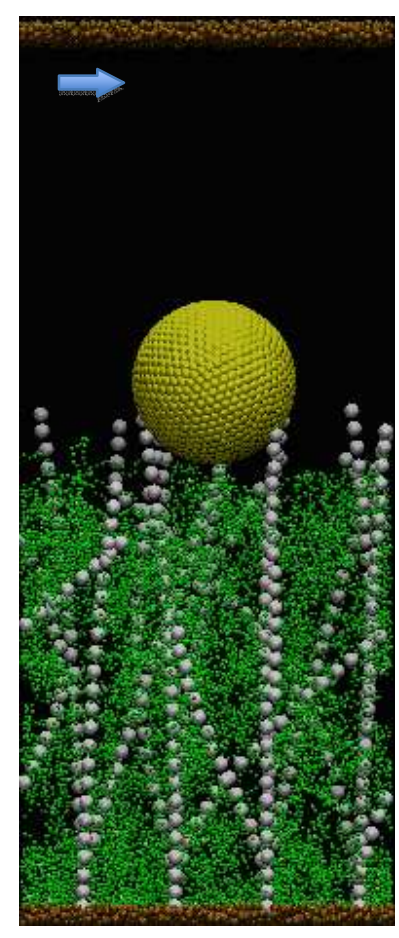

(b)

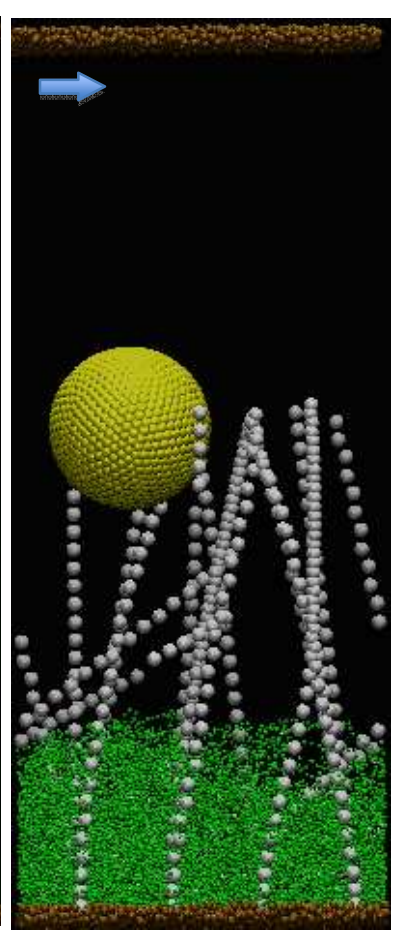

(c)

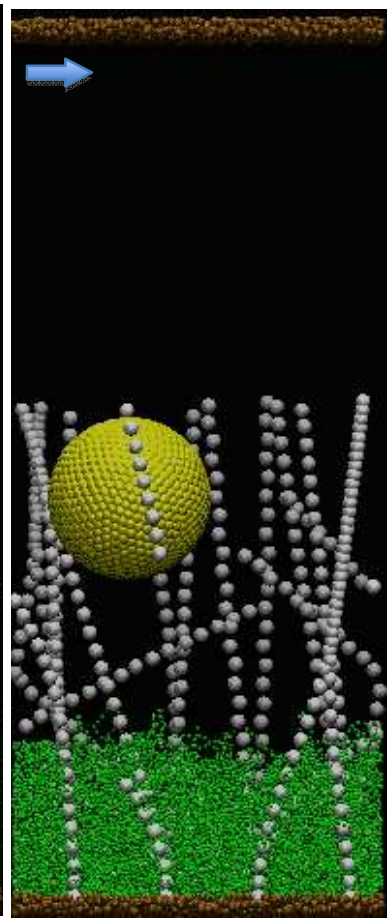

Figure 5 
(a)

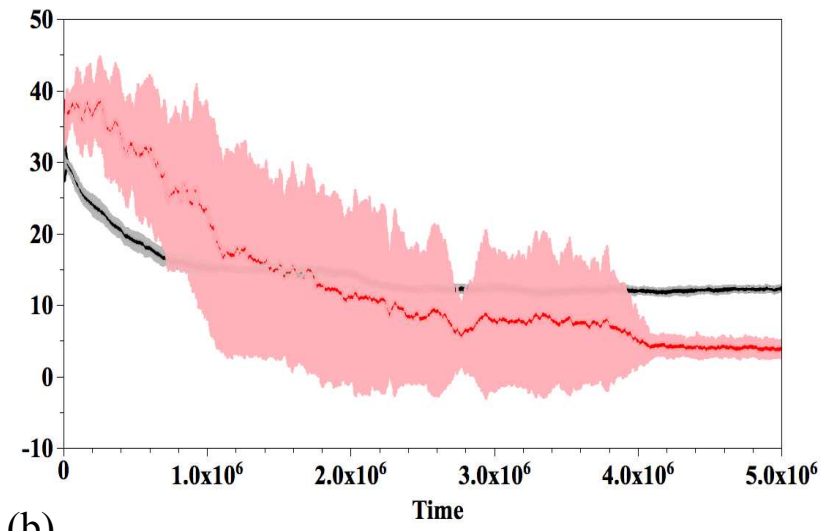

(b)

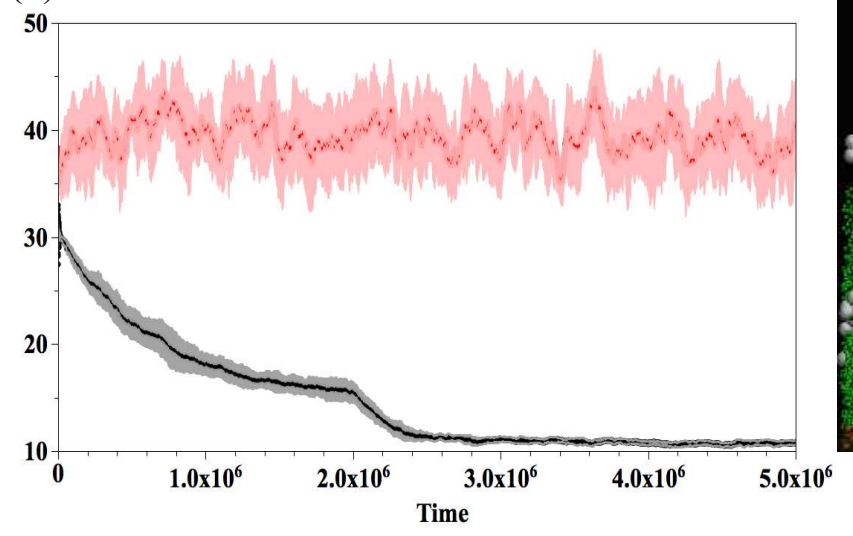

(c)

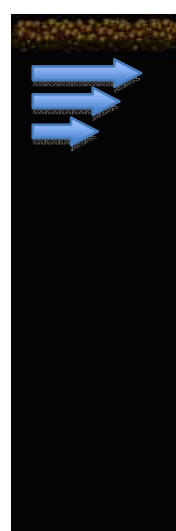

(d)

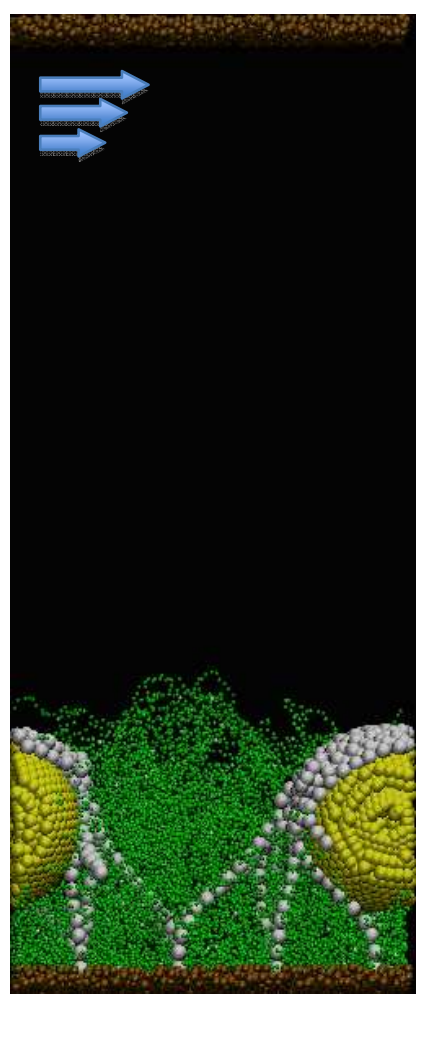

Figure 6 
Figure 7

(a)

(b)

(c)

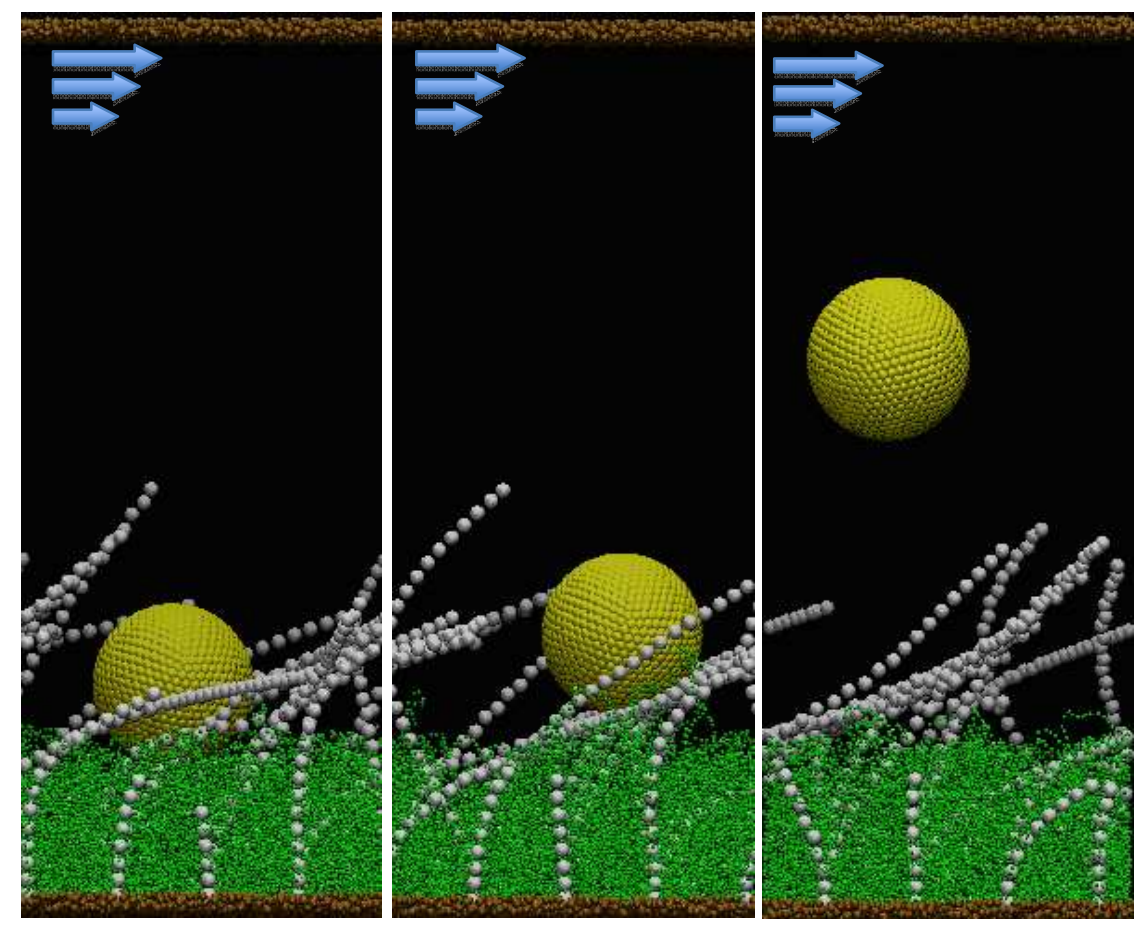




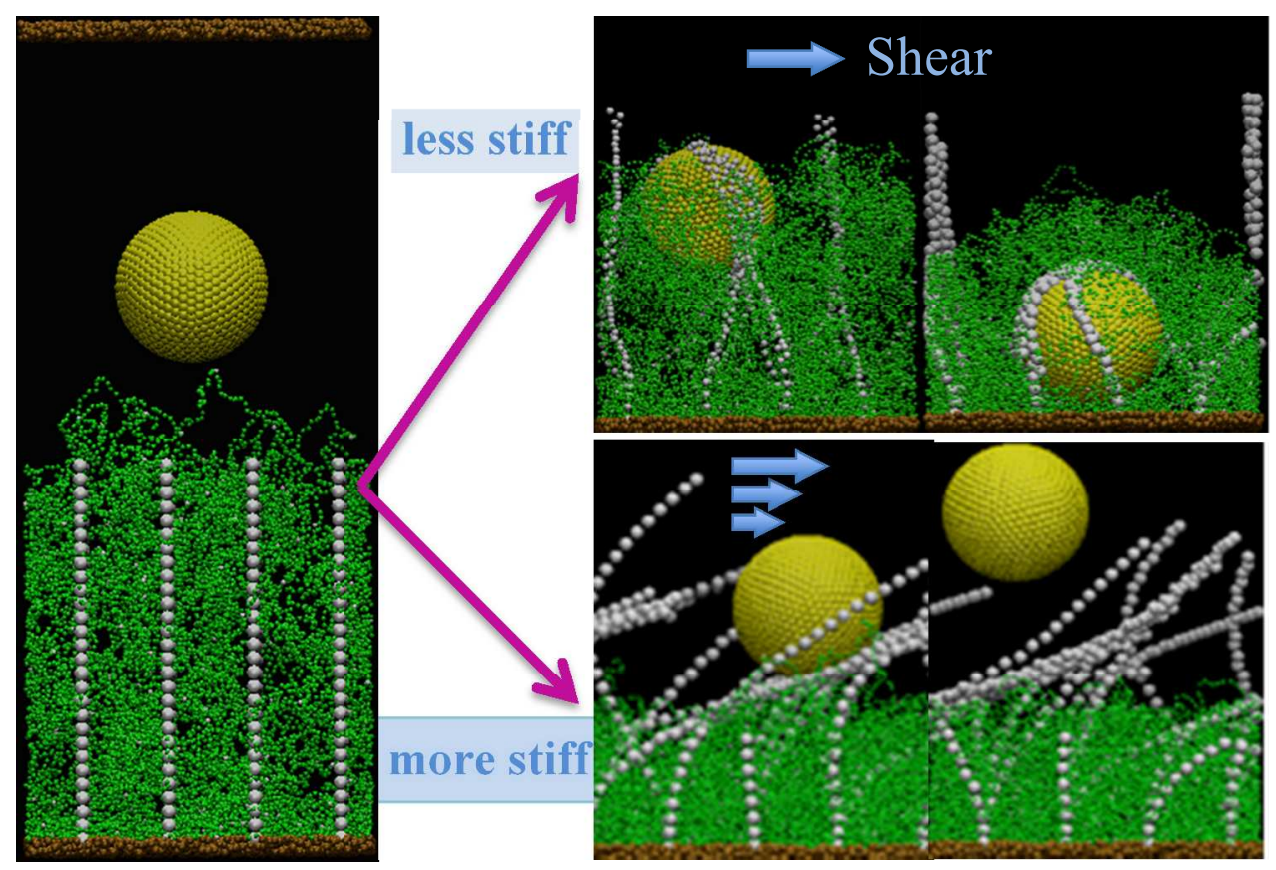

